\title{
O.S.P.
}

L'orientation scolaire et professionnelle

$47 / 1 \mid 2018$

L'héritage de Maurice Reuchlin

\section{Maurice Reuchlin et son histoire de la psychologie}

Maurice Reuchlin and his history of psychology

Serge Nicolas

\section{(2) OpenEdition}

Journals

Édition électronique

URL : http://journals.openedition.org/osp/5566

DOI : 10.4000/osp.5566

ISSN : 2104-3795

Éditeur

Institut national d'étude du travail et d'orientation professionnelle (INETOP)

Édition imprimée

Date de publication : 15 mars 2018

ISSN : 0249-6739

Référence électronique

Serge Nicolas, " Maurice Reuchlin et son histoire de la psychologie ", L'orientation scolaire et professionnelle [En ligne], 47/1 | 2018, mis en ligne le 01 mars 2020, consulté le 16 décembre 2020.

URL : http://journals.openedition.org/osp/5566 ; DOI : https://doi.org/10.4000/osp.5566

Ce document a été généré automatiquement le 16 décembre 2020.

(c) Tous droits réservés 


\title{
Maurice Reuchlin et son histoire de la psychologie
}

Maurice Reuchlin and his history of psychology

\author{
Serge Nicolas
}

1 Ma première « rencontre littéraire » avec Maurice Reuchin se fit d'abord par la lecture en 1984 de son "gros livre rouge» (ainsi que je l'appelais) intitulé simplement Psychologie (Reuchlin, 1984a) qui reste encore en bonne place dans ma bibliothèque personnelle. Recommandé par mes maitres à l'Université de Grenoble, il s'agissait de la cinquième édition (679 pages) du manuel de référence d'introduction à la psychologie de l'époque (la première édition, plus courte, datait de 1977). Le livre était riche et son contenu moderne, à l'époque, en ce qui concerne les problématiques de la psychologie scientifique, mais il n'y avait annexé aucun chapitre d'histoire de la discipline. Curieux d'en savoir plus sur les origines de la psychologie, je me procurai rapidement le petit «Que sais-je » du même auteur dans sa douzième édition (Reuchlin, 1984b). Publié pour la première fois en 1957 (voir figure 1), c'est dans ce livre que je découvris toute la richesse et la complexité historiques de la psychologie exposées dans une série de chapitres indépendants (vingtième édition en 2010). Comme ce petit livre est resté un classique français de l'histoire de la psychologie durant plus de 50 ans, il sera analysé dans la suite avec attention. Mais outre ce fameux "Que sais-je ", Reuchlin a aussi donné (et cela est moins connu) plusieurs autres textes traitant de l'histoire de la psychologie. 


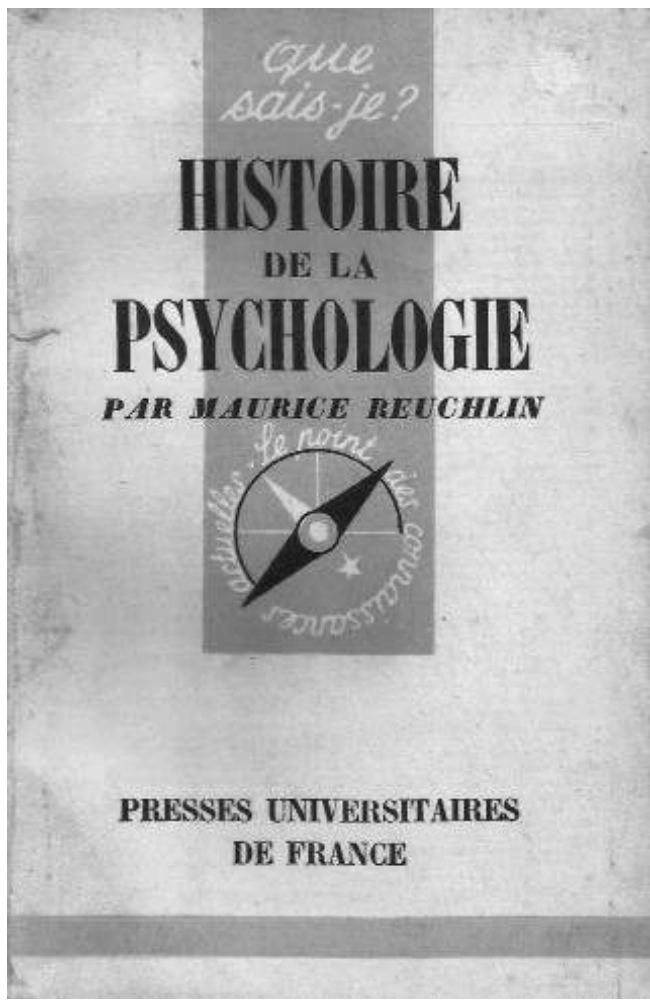

Figure 1. Cover of the first edition of the book of Reuchlin (1957)

2 L'intérêt de Reuchlin pour l'histoire de la psychologie date de ses premiers contacts avec la psychologie en tant qu'étudiant à Paris, comme il le souligne dans son autobiographie :

"J'avais éprouvé le besoin de situer dans le temps et si possible dans l'histoire des idées cette discipline si nouvelle pour moi [...] Cet intérêt se prolongea au-delà de ma période estudiantine. Me spécialisant en psychologie différentielle, j'essayai de mieux connaître les circonstances de son apparition, les polémiques qu'elle avait suscitées, les hommes qui la marquèrent de leur personnalité. Je m'intéressai beaucoup, en particulier, aux querelles soulevées par la psychophysique fechnérienne, et cet intérêt fut l'un des facteurs qui m'amenèrent à examiner de plus près la théorie de la mesure; je m'intéressai beaucoup aussi à la vie et à l'œuvre de Francis Galton [...]; et plus près de nous, à Alfred Binet dont je lus, je crois, toutes les publications. [...] J'avais alors une précieuse source d'information directe sur les psychologues du début du siècle, période cruciale pour le développement de la psychologie : les récits que me faisait Piéron de ses contacts et de ses activités à cette époque [...] Je consacrai à l'histoire de la psychologie plusieurs articles et, en 1957, mon premier livre. » (Reuchlin, 1992, p. 219).

Cependant, si l'on consulte la liste des écrits établis par Reuchlin (1991b) lui-même, on ne trouve guère de textes portant sur l'histoire de la psychologie avant 1957, si ce n'est un court article sur l'histoire de la psychologie appliquée (Reuchlin, 1955). Par conséquent, son ouvrage sur l'histoire de la psychologie constitue son principal écrit sur le sujet à cette époque. Mais la lecture de sa bibliographie (Reuchlin, 1991b) nous apprend qu'outre la rédaction de textes portant sur l'histoire de la psychologie différentielle (Reuchlin, 1970) et l'histoire de la psychologie appliquée (e.g. Reuchlin, 1971), il a également publié un article en anglais d'une grande importance, mais peu connu en France, portant sur l'histoire de la psychologie française (Reuchlin, 1965). 
C'est principalement sur la base des deux écrits généraux de Reuchlin $(1957,1965)$ que nous allons baser notre discussion sur la manière dont il concevait l'histoire de la discipline. Nous discuterons ainsi successivement du contenu de son ouvrage sur l'histoire de la psychologie (Reuchlin, 1957) ainsi que de l'article qu'il a consacré à l'histoire de la psychologie française (Reuchlin, 1965) publié dans le premier volume de la plus connue, encore aujourd'hui, des revues historiques du domaine (Journal of the History of the Behavioral Sciences).

\section{L'histoire de la psychologie vue par Maurice Reuchlin}

Comme le livre de Reuchlin (1957) sur l'histoire de la psychologie n'a subi que peu de changements, d'ailleurs souvent mineurs, au cours de ses vingt rééditions (entre 1957 et 2010 , voir figure 2) ${ }^{1}$, nous nous servirons principalement de l'édition originale pour appuyer nos propos, tout en indiquant, lorsque cela sera utile, les changements opérés d'une édition à l'autre.

Figure 2. Diverses couvertures du livre de Reuchlin sur l'histoire de la psychologie publié aux Presses Universitaires de France selon les années d'édition. A. 1966 ; B. 1984 ; C. 2003 ; D. 2012

[[Art2-fig2.j
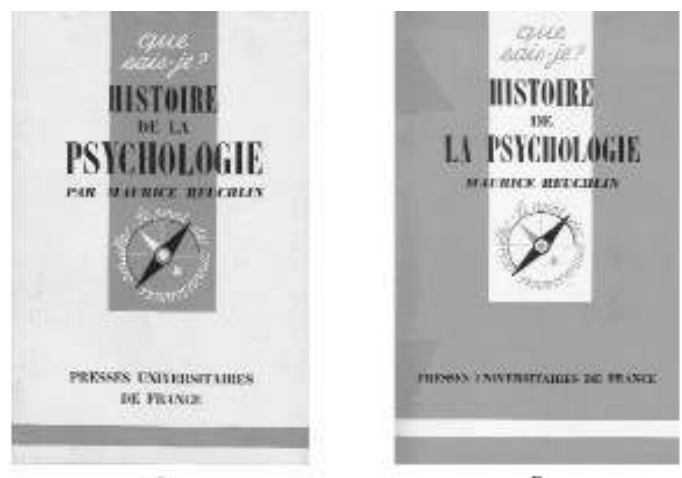

B
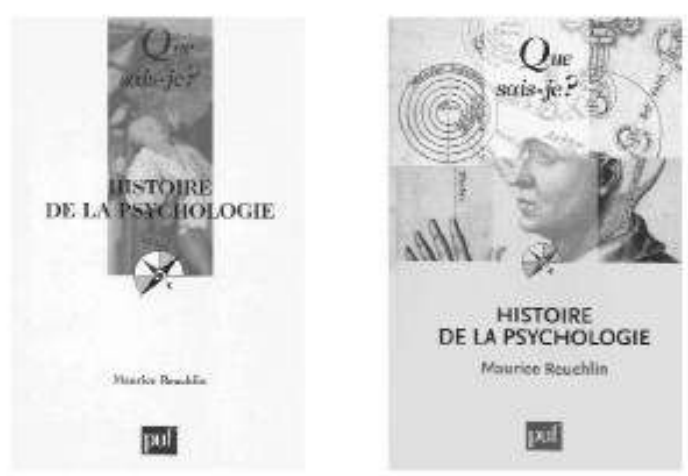

Figure 2. Diverse book covers of Reuchlin on the story of the psychology published in the University Press of France according to the years of publishing. A. 1966; B. 1984; C. 2003; D. 2012

\section{L'objectif de Reuchlin : promouvoir une histoire de la psychologie scientifique}

Le choix de l'exposé du petit livre (125 pages) de Reuchlin (1957) est clair : il nous donne une histoire de la psychologie nouvelle, celle qui est en train de se structurer en une discipline autonome. L'auteur se refuse à instituer une histoire des préoccupations 
philosophiques antérieures et prend le parti de traiter de la psychologie scientifique et de ses méthodes. Pour lui, la psychologie philosophique n'est pas une psychologie objective ; son caractère subjectif l'éloigne d'une forme de scientificité indispensable à l'établissement d'une discipline autonome. Il souligne à ce propos que «c'est vers l'accumulation de faits solidement établis dans un domaine limité que s'est [...] orientée la majeure partie des travaux scientifiques, en psychologie» (p.6). Pour Reuchlin, l'esprit de la recherche en psychologie ne s'est imposé que progressivement au cours du XIX ${ }^{e}$ siècle, et la familiarité avec les philosophies préparait mal à le comprendre. Mais l'auteur n'est pas un historien de la psychologie au sens moderne, car, comme il le dit lui-même, il s'appuie essentiellement sur des données de seconde main pour développer son propos. Ainsi, il se réfère surtout à des ouvrages historiques américains, notamment au livre de Boring (1950), dont le contenu lui fournit des informations importantes comme il le confesse dans les premières éditions de son "Que sais-je?" (Reuchlin, 1957, 1984b) et dans son autobiographie: "Je m'étais attelé à l'étude de l'histoire de la psychologie en lisant attentivement le gros livre d'E. G. Boring" (Reuchlin, 1992). Il est à noter que l'emprunt qu'il fait à Boring n'est cependant plus mentionné dans son histoire de la psychologie à partir de la quinzième édition de 1991 (Reuchlin, 1991a).

7 Reuchlin (1957) justifiait son approche sous-disciplinaire de l'histoire de la psychologie en soulignant qu' i il est légitime de distinguer aujourd'hui des domaines différents » (p.6). De fait, le livre sera structuré en six chapitres historiques distincts: la psychologie expérimentale, la psychologie animale, la psychologie différentielle, la psychologie pathologique et la méthode clinique, la psychologie de l'enfant, la psychologie sociale. Il n'est pas dans mon intention de faire ici une présentation exhaustive de l'ouvrage, mais plutôt de centrer mon propos tour à tour sur trois chapitres parmi les plus emblématiques: l'histoire de la psychologie expérimentale, l'histoire de la psychologie pathologique et clinique, l'histoire de la psychologie différentielle.

\section{L'histoire de la psychologie expérimentale}

$8 \quad$ Le livre de Reuchlin (1957) débute par la psychologie expérimentale qui y est traitée au plan historique dans le premier chapitre, le plus long du livre (pp. 9-31). La psychologie expérimentale y est considérée par l'auteur comme une psychologie générale : «elle recherche des "lois" valables pour l'espèce humaine tout entière, voire pour l'ensemble des êtres vivants » (p.43). Reuchlin débute cette histoire en montrant que les progrès de la psychologie sont d'abord la conséquence du développement de la physiologie, notamment la physiologie sensorielle (d'où le fait que les premiers psychologues étaient souvent des physiologistes). «On éprouve toujours une certaine difficulté à délimiter de façon précise les domaines respectifs de la physiologie et de la psychologie: l'existence d'une discipline intermédiaire, la psychophysiologie en témoigne" (p.10). Parmi les pionniers présentés dans ce chapitre, on trouve notamment les noms de Gustav Theodor Fechner (1801-1887) et Hermann von Helmholtz (1821-1894). Mais c'est à Wilhelm Wundt (1832-1920) que Reuchlin (pp.16-19) attribue définitivement, et avec raison, l'autonomie de la psychologie expérimentale. Par ses travaux expérimentaux, son laboratoire fondé en 1879 (Nicolas, 2005b) et son centre de formation (l'Institut de psychologie de Leipzig), Wundt est incontestablement le personnage qui a marqué de son empreinte l'établissement de la 
nouvelle psychologie (scientifique et objective). Mais, dans son texte, Reuchlin souligne à tort que Wundt a mis à l'honneur la méthode d'introspection, alors qu'il est celui qui l'a combattue avec le plus de force dans de retentissants articles (Wundt, 1907, 1908). Reuchlin poursuit son texte en montrant les influences ultérieures de ces pionniers sur les travaux d'Ebbinghaus (1850-1909) portant sur la mémoire et ceux de l'école de Würzburg sur la pensée. C'est dans ce contexte que sont rapidement évoqués (pp. 20-21) les premiers travaux expérimentaux d'Alfred Binet (1857-1911) sur la mémoire des idées et la psychologie individuelle. Reuchlin poursuit en présentant l'évolution de la psychologie expérimentale à travers ses écoles : l'école allemande de la Gestalt (Köhler, Koffka, Wertheimer), l'école objectiviste russe (surtout Pavlov; le nom de Bechterew n'étant mentionné qu'en passant) et américaine (béhaviorisme) avec notamment la figure de John Broadus Watson (1878-1958) anticipé selon l'auteur par Piéron en 1908 (alors qu'en réalité l'attitude objective était déjà prépondérante chez de nombreux psychologues américains et allemands de l'époque). Çà et là dans le texte, on trouve quelques éléments d'histoire de la psychologie expérimentale française qui permettent de définir le rôle de certains psychologues français dans ce mouvement. Même si l'ouvrage a été réactualisé en 1991 et en 2003, le mouvement cognitiviste n'est évoqué qu'en passant, ce qui ne permet pas au lecteur de se faire une idée précise sur le mouvement actuel de la recherche dans ce domaine.

\section{L'histoire de la psychologie différentielle}

Après une présentation rapide de l'histoire de la psychologie animale dans le deuxième chapitre (pp.32-42), le chapitre suivant porte sur l'histoire de la psychologie différentielle (pp. 43-59). Spécialiste du domaine, Reuchlin souligne que la psychologie différentielle n'est pas une psychologie générale. La psychologie différentielle s'oppose à la psychologie générale expérimentale en ce que «la "loi", la relation, vraie dans sa forme générale pour l'espèce entière, se diversifie entre certaines limites lorsqu'on considère des individus ou des groupes différents. L'étude de ces variations entre individus ou entre groupes constitue l'objet de la psychologie différentielle» (p. 43). Pour Reuchlin, le fondateur de cette orientation de recherche est sans nul doute le savant anglais, cousin de Charles Darwin, Francis Galton (1822-1911) par ses travaux empiriques sur les problématiques d'hérédité et de mesure (tests) ainsi que par ses travaux mathématiques sur les corrélations, poursuivis par ses disciples qui vont développer une psychologie statistique très influente (pour des développements ultérieurs : Reuchlin, 2003a, 2003b, 2003c). Dans cette première section du chapitre III, plusieurs pages du livre sont ainsi consacrées à Galton, mais aussi aux statisticiens et psychologues anglais Karl Pearson, Charles Spearman, et Cyril Burt. Les psychologues anglais qui ont utilisé l'analyse factorielle afin de décrire le comportement humain par l'existence de facteurs ont nettement favorisé une conception hiérarchique de l'intelligence. La deuxième section du chapitre, traitant des théories relatives aux différences individuelles, est précédée (dans la cinquième édition du "Que sais-je?", Reuchlin, 1966) par la mention de l'existence d'un tout nouveau laboratoire de psychologie différentielle créé à l'EPHE (École pratique des hautes études) en 1964 sous la direction de Reuchlin (Reuchlin, 2001). Comme les différences constatées entre les individus sont dues à la fois à des causes constitutionnelles liées à l'hérédité et à l'influence de l'environnement, elles doivent être en partie modifiables par une action du milieu. Reuchlin souligne parfaitement que « des désaccords notables existent sur 
l'importance respective des deux facteurs, sur le mécanisme de leur action et par suite, sur les limites des possibilités transformatrices du milieu » (p. 50); mais bien peu de données historiques sont fournies au lecteur. C'est dans la dernière section du chapitre traitant du développement des applications de la psychologie différentielle que l'on trouve un court historique sur les tests d'intelligence où l'auteur souligne que c'est Binet et Henri (1896) qui ont montré dans leur article sur la psychologie individuelle, après les échecs des tentatives américaines de Cattell et de Jastrow, que la meilleure façon de différencier les individus consiste à le faire sous l'angle de leurs facultés supérieures. Il souligne que, suite à une demande ministérielle liée à la mise en place de classes spécialisées, Binet et Simon (1905) vont proposer la première version de leur échelle métrique d'intelligence dans le but de pouvoir recruter les enfants en retard intellectuel (pour un historique récent : Nicolas, Andrieu, Croizet, Sanitioso, \& Burman, 2013). Le chapitre se termine par un court historique sur la psychologie différentielle en France où l'on trouve entre autres les noms d'Édouard Toulouse (1865-1947), de Jean-Maurice Lahy (1872-1943) et de Dagmar Weinberg (1897-1946). Mais aucun historique n'est fourni sur cette question pour d'autres pays, notamment l'Allemagne et les États-Unis, où la psychologie différentielle et les techniques de sélection scolaire et professionnelle ont été cultivées avec ardeur (pour un historique ultérieur: voir Reuchlin, 1970). Nulle mention n'est aussi fournie dans les éditions suivantes de l'ouvrage sur les liens forts existant aujourd'hui entre la psychologie différentielle et la psychologie cognitive expérimentale.

\section{L'histoire de la psychologie pathologique}

10 Comme le note Reuchlin (p.60), la psychologie pathologique est une psychologie essentiellement d'origine française. De fait, l'auteur présente dans ce chapitre une véritable histoire de la psychologie française, en montrant l'importance de la psychiatrie pour la psychologie. Reuchlin va donner une place centrale, en y consacrant la première section du chapitre (quatre pages entières), au personnage qui a fondé la psychologie française moderne : Théodule Ribot (1839-1916). Il voit avec raison en Ribot une figure majeure de la psychologie française (voir Nicolas, 2005a) qui a déclaré l'autonomie de la psychologie vis-à-vis de la philosophie et de la physiologie. En effet, le philosophe et psychologue Ribot va mettre en place la méthode pathologique en psychologie, basée sur l'identité fondamentale des mécanismes normaux et pathologiques, en s'appuyant sur les écrits évolutionnistes d'Herbert Spencer (1820-1903) et neurologiques de John H. Jackson (1835-1911). Pour Ribot, c'est sous l'angle de la pathologie que l'on peut étudier les grandes fonctions mentales, car la pathologie provoque une régression, une dissolution des fonctions mentales et en fait apparaître les mécanismes cachés. Un exemple majeur de la fécondité de cette approche va être fourni par Ribot (1881) dans le domaine de la mémoire où il va déduire sa loi de régression selon laquelle, dans les traumatismes et la démence sénile, ce sont d'abord les souvenirs les plus récents, mal fixés au niveau organique, qui sont affectés en premier. Cette méthodologie de la recherche, axée sur l'étude des pathologies, va être poursuivie par ses élèves Pierre Janet (1859-1947) et Georges Dumas (1866-1946), auxquels Reuchlin consacre la deuxième section de son chapitre. Il souligne que l'apport de Janet fut cependant plus original à l'égard de leur maître commun que ne le fut celui de Dumas. La thèse de Janet sur l'automatisme psychologique atteste de ce fait (Janet, 1889). Janet, qui s'est placé résolument sur le 
plan psychologique, a opposé deux activités fondamentales de l'esprit: l'activité de synthèse (la conscience) et l'activité conservatrice (l'automatisme). Ces deux activités subsistent ordinairement ensemble. Quand l'esprit est normal, il n'abandonne à l'automatisme que certains actes inférieurs. Quand l'esprit n'est plus normal, on trouve une prédominance de l'automatisme ancien sur une activité synthétique actuelle très affaiblie. Reuchlin ne parle pas dans son texte du subconscient de Janet, mais préfère évoquer les conceptions tardives de Janet sur la notion de conduite, en montrant que c'est l'action qui est à l'origine de la pensée. Les premières recherches de Janet avaient porté sur la question de l'hystérie et de l'hypnose, ces travaux avaient été initiés au siècle précédent par Mesmer (1734-1815) puis développés par Jean-Martin Charcot (1825-1893) auquel Reuchlin consacre la troisième section de son chapitre même s'il considère que l'histoire de l'école de la Salpêtrière intéresse plus directement la médecine que la psychologie.

«Mais elle ne peut être passée sous silence. D'abord sans doute parce qu'elle fut fréquentée par des psychologues comme P. Janet, A. Binet ou S. Freud. Mais aussi peut-être parce que les travaux qui s'y sont déroulés sur la suggestion et l'hypnotisme chez les hystériques se rattachent à un courant de préoccupations dont les manifestations en "psychologie dynamique" sont importantes » (p. 69).

11 Après avoir retracé l'historique de cette question, il aborde dans la quatrième section la psychanalyse de Freud sans évoquer les autres courants psychanalytiques. Le chapitre se termine par un exposé de la méthode clinique inventée en 1896 par un obscur élève de Wundt, Lightner Witmer (1867-1956), et popularisé en France par Daniel Lagache (1903-1972). Reuchlin montre que l'histoire de la psychologie clinique est dominée par la présence de deux attitudes: 1) examen historique, exhaustif, aboutissant à un tableau du cas et à un pronostic nuancé ; 2) examen par tests, dans des conditions standardisées, aboutissant à des profils ou à des pronostics sous forme numérique.

"Lorsqu'on parle alors de "méthode clinique", c'est à la première de ces deux attitudes que l'on pense. On la rattache parfois à une "tradition dynamique" issue de Ribot, Janet et Freud, alors que la seconde attitude appartiendrait à la "tradition psychométrique" de Wundt et Galton... Les deux attitudes semblent correspondre à deux formes d'esprit, à deux types d'intérêt différents, et les psychologues qui sont attirés fortement par l'une d'elles ne le sont guère, en général, par l'autre " (pp. 81-82).

Reuchlin souligne en passant que le succès de Binet provient certainement pour une large part de la synthèse qu'il parvint à réaliser entre ces deux méthodes.

On voit qu'à la lecture de ces divers chapitres ${ }^{2}$ Reuchlin parle de Binet à divers endroits de son texte, mais sans le présenter comme une figure centrale de la psychologie puisque aucune bio-bibliographie ne lui est consacrée dans le livre (comme il l'a fait notamment pour Fechner, Wundt, Galton, Ribot, Janet, Dumas, Freud, etc.). Est-ce à dire qu'il ne considérait pas le personnage comme une figure de niveau international ? Probablement, mais on pouvait alors s'attendre à ce que Binet apparaisse en bonne place dans une histoire de la psychologie française.

\section{L'Histoire de la psychologie française vue par Maurice Reuchlin} Reuchlin (2002) me faisait l'insigne honneur d'en écrire la préface. Il y louait 
notamment mon attitude objective en tant qu'historien, contrastant avec celle d'autres spécialistes de la discipline qui, selon lui, privilégiaient une perspective plus subjective. Entre objectivité et subjectivité, que dire alors du traitement historique qu'a donné Reuchlin de l'œuvre si riche de Binet (voir Nicolas \& Levine, 2012)?

\section{Alfred Binet vu par Piéron et les psychologues français}

Lorsque dans son autobiographie Reuchlin (1992) parle de Binet, il écrit avoir lu (et on peut le croire) toutes ses publications et souligne : «elles nous laissent apparaître de façon vivante une personnalité attachante par ses contradictions» (p. 219). Ces contradictions, dont Reuchlin a longuement parlé dans un article sur Binet (Reuchlin, 1958), étaient celles d'un chercheur honnête et compétent, et celles d'un scientifique ouvert aux nouveautés et aux diverses méthodes de la psychologie. Cependant, Binet a subi toute sa vie des critiques personnelles et une mise à l'écart de la part de ses compatriotes, dont Ribot et Piéron, qui ont toujours minimisé son apport à la psychologie nouvelle ${ }^{3}$ (pour un historique, voir Nicolas, Andrieu, Croizet, Sanitioso, \& Burman, 2013). "Nul n'est prophète dans son pays ", c'est un adage que Binet s'attribuait à lui-même (voir Nicolas, Gounden, \& Sanitioso, 2014). On pourrait parler longuement des causes de ce rejet, mais pour ma part, j'incline à penser que Binet était une personnalité scientifique atypique dans le paysage psychologique français: il n'avait ni une formation classique pour un psychologue de l'époque (Nicolas \& Ferrand, 2002) ni une activité sociale communicante (il détestait les Congrès et considérait que ce n'était là que de vaines parlottes). En fait, Binet était une figure originale souvent incomprise, et comme l'a écrit Piéron (1912) dans sa nécrologie :

« Les honneurs n'avaient aucun attrait pour lui et ne lui en imposaient aucunement.

[...] Aussi la société ne rendit pas pleinement justice à cet indépendant trop personnel et trop critique, qui ne savait pas ce que c'est que de plier, et peut-être même avait une tendance spontanée à l'opposition, à la contradiction, tant il sentait la nécessité de résister et de combattre au nom de la logique et de la raison. S'il n'occupa point dans les hiérarchies officielles le rang qui aurait dû lui revenir, Alfred Binet représenta le mieux, aux yeux de l'étranger ${ }^{4}$, la psychologie scientifique française dont il fut un des premiers et un des plus fidèles pionniers » (p. 245).

16 Ainsi, Binet est présenté par Piéron comme un psychologue touche à tout, critique et entêté, dont les idées étaient souvent en opposition avec celles de ses contemporains sur bien des points. Mais Piéron ne peut que constater la stature internationale acquise par Binet, alors qu'il n'avait pas perçu lui-même la formidable nouveauté de l'échelle métrique d'intelligence lorsqu'elle fut publiée pour la première fois en 1905. Mais Piéron est forcé, dans sa nécrologie, d'être le laudateur de Binet :

« En somme, Binet fut surtout, suivant l'expression de Claparède, "un découvreur"; il se sentit attiré de trop de côtés pour se livrer exclusivement à une catégorie de recherches, s'acharner à approfondir un unique sillon. À coup sûr, certains de ses travaux ne sont pas définitifs, certaines de ses idées sont discutables, et comment en serait-il autrement dans des domaines nouveaux et presque inexplorés ? Mais bien des faits établis avec cette conscience, cette honnêteté scientifique si précieuse qui le caractérisaient, sont aujourd'hui acquis ; bien de ses suggestions ouvrirent des voies nouvelles à l'étranger, car il eut peu d'élèves en France. La psychologie française, qui ne peut opposer que bien peu de noms à la cohorte des savants allemands ou américains, perd en Alfred Binet un de ceux qui lui ont le plus fait honneur » (pp. 245-246). 
17 Mais il n'est pas certain que tout cela corresponde aux sentiments de Piéron qui ne considérait pas Binet comme un véritable scientifique. La correspondance qui nous est parvenue entre Piéron et Binet (voir Klein, 2011) est, selon les termes mêmes de Klein (2016), «une relation essentiellement professionnelle, toujours polie, mais certainement pas exempte pour autant de certaines tensions » (p. 28). Reuchlin, qui a appris beaucoup de choses au cours de ses conversations avec Piéron (Reuchlin, 1992, p. 219), a certainement été influencé par l'avis de son maître qui entretenait un profond désaccord avec Binet au plan épistémologique. Comme le note Klein (2016), Piéron et Binet adhéraient à deux modèles épistémologiques bien distincts sur la question de la psychologie scientifique. Pour Binet, la psychologie est une science de l'homme; elle doit concilier le quantitatif (psychologie expérimentale) et le qualitatif (psychologie subjective). Au contraire, pour Piéron, adhérant à un réductionnisme strict, la psychologie est sous-tendue par la physiologie; elle doit être une psychophysiologie dont la méthode est purement expérimentale (Nicolas \& Gounden, 2016). Ainsi, pour Piéron et pour Reuchlin, la psychologie de Binet est une psychologie surannée, appartenant à un passé où ses bases scientifiques (expérimentales) n'étaient pas encore fermement établies.

\section{Le traitement par Reuchlin de la psychologie de Binet}

Cette psychologie de Binet était tellement considérée comme passéiste et secondaire que lorsque Reuchlin présente en août 1963 une communication sur l'histoire de la psychologie française au cours $\mathrm{du} \mathrm{XVII}^{\mathrm{e}}$ Congrès international de psychologie à Washington, il ne parle de l'œuvre et de l'influence de Binet qu'en passant. Cette communication fut publiée sous forme d'article (Reuchlin, 1965) dans le premier volume du tout nouveau Journal of the History of the Behavioral Sciences, la plus ancienne et encore aujourd'hui la plus influente revue d'histoire de la psychologie. Reuchlin débute son article en présentant d'abord le fondateur de la nouvelle psychologie française: Théodule Ribot (Nicolas, 2005a; Nicolas \& Murray, 1999). Il donne une histoire de la psychologie en France en partant de la période commençant par l'accession en 1888 de Ribot à la chaire de psychologie expérimentale et comparée du Collège de France (Nicolas \& Charvillat, 2001) jusqu'à la prise de fonction de Piéron en 1912 au laboratoire de psychologie physiologique de la Sorbonne en remplacement de Binet (Nicolas, Gras, \& Segui, 2011). À la lecture du texte de Reuchlin, on mesure avec raison la grande influence de Ribot sur la psychologie de l'époque et l'émergence de ceux qui se sont réclamés de lui, notamment Pierre Janet et Georges Dumas qui seront choisis pour succéder à Ribot, l'un au Collège de France, l'autre à la Sorbonne, tout cela au détriment de Binet (Nicolas \& Ferrand, 2002). Binet n'est encore signalé dans ce nouveau texte de Reuchlin qu'en passant; cela a dû paraître étonnant aux lecteurs américains. La deuxième partie de l'article traite de l'accession de Piéron en tant que leader de la psychologie française d'après-guerre. Une grande partie du texte lui est consacrée ainsi qu'à son influence institutionnelle au cours de cette période. Piéron y est décrit comme un (psycho)-physiologiste, dont les compétences ont été reconnues par le Collège de France où il accède en 1923 à la chaire de physiologie des sensations (Nicolas, Gyselinck, \& Piolino, 2015). Bien d'autres psychologues sont cités pour cette période d'entre-deux-guerres, notamment Pierre Janet, Georges Dumas, Jean-Maurice Lahy, Paul Guillaume, Henri Wallon et Jean Piaget. L'article de Reuchlin se termine par les tendances de la période récente qui indiquent nettement un 
développement considérable des recherches à caractère non physiologique; les noms de Paul Fraisse, René Zazzo, Pierre Oléron, Daniel Lagache, Juliette Favez-Boutonnier sont notamment cités (pour une présentation historique de tous ces personnages, voir Nicolas, 2002). Ainsi, la figure de Binet est-elle noyée dans une histoire dominée, aux yeux de Reuchlin, par les grands maîtres de la psychologie française que sont Ribot et Piéron. Reuchlin participera ainsi plus ou moins volontairement, mais il ne sera pas le seul jusqu'à récemment, à la méconnaissance de l'œuvre incroyablement riche, diverse et novatrice de Binet que l'auteur de cet article et son groupe cherche aujourd'hui à réhabiliter (pour des publications récentes : Nicolas, 2015, 2016 ; Nicolas \& Guida, 2016 ; Nicolas \& Makowski, 2016; Nicolas, Andrieu, Sanitioso, Vincent, \& Murray, 2015 ; Thomas, Didierjean, \& Nicolas, 2016).

\section{Conclusion : une histoire " personnelle » de la psychologie}

19 L'article américain de Reuchlin (1965) sur la psychologie française est peu connu, du moins en France, ce qui n'est pas le cas de son livre maintes fois réédité sur l'histoire de la psychologie (Reuchlin, 1957, 2010). Lors de sa parution, le livre de Reuchlin (1957) fut très bien accueilli par un collaborateur éminent de L'Année psychologique :

« Il n'existait pas en France d'histoire de la psychologie avant ce petit livre qui vient donc à point combler une lacune. À juste titre l'auteur s'est limité à l'histoire de la psychologie scientifique, soit en gros à un siècle de développement [...] Ce n'est pas le moindre mérite de $\mathrm{M}$. Reuchlin que de s'attacher plus à l'évolution des problèmes et des techniques qu'aux faits anecdotiques - en limitant son champ d'étude il l'a approfondi et est parvenu à donner un texte aussi exhaustif que la taille de l'ouvrage pouvait le permettre. En évitant l'écueil d'écrire un abrégé des ouvrages de référence tels que ceux de Boring, $M$. Reuchlin a écrit un livre excellent et utile » (Bresson, 1957, p. 555).

Il est vrai que Reuchlin s'était surtout donné pour but d'intéresser ses lecteurs à l'histoire de la psychologie en leur fournissant une documentation pédagogique qui faisait défaut à l'époque. Certes ce livre avait également le mérite de proposer une histoire sous-disciplinaire de la psychologie axée essentiellement sur l'importance jouée par certains pionniers de la discipline. Mais cette approche sera critiquée ultérieurement par certains historiens français de la psychologie. Par exemple, Ohayon (1999) écrit :

«En 1957, paraît le premier "Que sais-je ?" consacré à l'Histoire de la psychologie. Rédigé par Maurice Reuchlin, il met à mal "L'unité de la psychologie" de Lagache, en privilégiant sérieusement l'approche expérimentale. Un modèle pour l'histoire de la psychologie se construit alors, celui de la rupture opérée par des pionniers (Wundt, Ribot) avec la philosophie et l'introspection, pour construire une discipline scientifique en s'appuyant sur la physique et la physiologie » (p. 401).

Même si d'autres modèles plus pertinents de présentation de l'histoire de la psychologie sont possibles (Jalley, 2007), force est de constater que certains personnages ont tout de même donné une impulsion majeure au développement de la psychologie en tant que science autonome. Ce sont notamment les ruptures proposées par Wundt, Ribot, Galton et par bien d'autres qui ont permis l'émergence de la discipline. De plus, comme le souligne Jalley (2007): "L'ouvrage de Reuchlin [...] convenait tout à fait bien à une époque où l'enseignement universitaire de la 
psychologie se calait déjà sur un parti pris de schématisation soi-disant scientifique [...] et où s'amorçait aussi la lutte féroce, au sein de la discipline entre les "scientifiques" et les littéraires"(pp. 89-90). Comme la psychologie est encore largement enseignée aujourd'hui de manière compartimentée (psychologie cognitive expérimentale, psychologie différentielle, psychologie sociale, etc.), le livre de Reuchlin a eu un succès considérable et demeure encore aujourd'hui d'une utilité pratique évidente (le livre n'est malheureusement plus réédité, un autre ouvrage qui propose une histoire plus intimement liée à celle de la philosophie l'a remplacé dans la même collection (Houdé, 2016). Cependant, force est de constater que Reuchlin, qui a « joué l'historien » comme il l'écrit lui-même (Reuchlin, 1957, p. 124), fait une présentation quelque peu tronquée de l'évolution historique de la psychologie par les carences que l'on peut aisément constater (voir Jalley, 2007) et en mettant plus l'accent sur l'importance de la psychologie française tout en négligeant l'apport au plan théorique et au plan scientifique des psychologies allemandes et surtout américaines. S'il est vrai qu'il était tout à fait légitime de faire la promotion de la psychologie française dans un «Que saisje? ", on peut néanmoins reprocher à Reuchlin de n'avoir pas présenté de manière plus étendue l'évolution historique des grandes orientations théoriques de la psychologie. Il entreprendra cependant une telle démarche quelques années plus tard avec la publication de son ouvrage "Totalités, éléments, structures en psychologie» (Reuchlin, 1995) où l'histoire de la psychologie y occupe une place importante.

Cette écriture "personnelle " de l'histoire de la psychologie me semble bien illustrée par la négligence voulue (et coupable) avec laquelle Reuchlin parle du psychologue français le plus emblématique et le plus célèbre à l'étranger : Alfred Binet, bien que son nom soit cité à divers endroits du livre. Cette négligence est-elle accidentelle ou intentionnelle? Nous avons pu répondre à cette question en présentant le contenu du rapport qu'a donné Reuchlin en 1965 sur l'histoire de la psychologie française. L'analyse d'autres écrits historiques de Reuchlin $(1955,1970,1971,1995)$ aurait conduit à la même conclusion: une sous-estimation intentionnelle de l'importance et de l'influence de l'œuvre de Binet, notamment en psychologie différentielle (Reuchlin, 1970) et en psychologie appliquée (Reuchlin, 1971). Ainsi, les récits de l'histoire de la psychologie peuvent être multiples et, comme l'écrivent Carroy, Ohayon et Plas (2006, pp. 237-238): «L'historien a en effet le choix entre deux types de récit. Il peut écrire l'histoire d'une discipline en partant du présent et des vocables actuels (Reuchlin, 1957 ; Nicolas, 2002). Il essaie alors d'identifier des précurseurs et il laisse plus ou moins de côté la singularité d'une époque. Pour démontrer son caractère scientifique, il accentue la proximité de la psychologie et des sciences de la vie. L'historien peut adopter un autre point de vue et tenter d'écrire l'histoire transdisciplinaire d'une ou plusieurs psychologies inscrites dans la culture et dans les sciences humaines... ». Contrairement à Carroy et al. (2006), nous pensons que la psychologie contemporaine a bénéficié de l'avancée des sciences et des techniques au cours du xix siècle et, de fait, elle a besoin d'être légitimée par une quête de précurseurs qui ont été sensibles à ces changements car ils représentent par leurs idées, leur attitude, leur engagement et leurs travaux les ruptures qui ont permis l'émergence d'une psychologie autonome. Reuchlin a eu raison dans ses écrits de rechercher et mettre en avant l'importance des précurseurs, mais il aurait dû beaucoup plus insister sur le fait que Binet fut l'un de ceux-là, et certainement l'un des plus importants ! 


\section{BIBLIOGRAPHIE}

Binet, A., \& Henri, V. (1896). La psychologie individuelle. L'Année psychologique, 2, 411-465.

Binet, A., \& Simon, T. (1905). Méthodes nouvelles pour le diagnostic du niveau intellectuel des anormaux (travaux de l'année 1904). L'Année psychologique, 11, 191-244.

Boring, E. G. (1950). A history of Experimental Psychology. New York: Appleton.

Bresson, F. (1957). Analyse de l'ouvrage de M. Reuchlin, Histoire de la psychologie, In $8^{\circ}$ de 125 pages, «Que sais-je? », Paris : Presses Universitaires de France, 1957. L'Année psychologique, $57,555$.

Carroy, J., Ohayon A., \& Plas, R. (2006). Histoire de la psychologie en France. Paris : La Découverte. Houdé, O. (2016). Histoire de la psychologie (coll. « Que sais-je ? »). Paris : Presses Universitaires de France.

Jalley, E. (2007). Critique de la raison en psychologie. La psychologie scientifique est-elle une science? Paris : L'Harmattan.

Janet, P. (1889). L'Automatisme psychologique. Essai de psychologie expérimentale sur les formes inférieures de l'activité humaine. Paris : F. Alcan.

Klein, A. (éd.) (2011). Correspondance d'Alfred Binet (vol. II). Nancy : Presses Universitaires.

Klein, A. (2016). Henri Piéron, élève et digne successeur d'Alfred Binet ? In L. Gutierrez, J. Martin \& R. Ouvrier-Bonnaz (éd.), Henri Piéron (1881-1964), Psychologie, Orientation et Éducation (pp. 25-38). Toulouse : Octarès.

Nicolas, S. (2002). Histoire de la psychologie française. Paris : In Press.

Nicolas, S. (2005a). Théodule Ribot. Philosophe breton fondateur de la psychologie française. Paris : L'Harmattan.

Nicolas, S. (2005b). Wundt et la fondation en 1879 de son laboratoire. Histoire documentaire de la création et du développement de l'Institut de psychologie expérimentale de Leipzig. L'Année psychologique, 105, 133-170.

Nicolas, S. (2015). Psychology in French academic publishing in the late 19th century: Alfred Binet, editorial director at the Schleicher publishing house. Journal of the History of the Behavioral Sciences, 51(3), 285-307.

Nicolas, S. (2016). The importance of instrument makers for the development of experimental psychology: the case of Alfred Binet at the Sorbonne laboratory. Journal of the History of the Behavioral Sciences, 52(2), 231-257.

Nicolas, S., \& Charvillat, A. (2001). Introducing psychology as an academic discipline in France: Théodule Ribot and the "Collège de France"(1888-1901). Journal of the History of the Behavioral Sciences, 37, 143-164.

Nicolas, S., \& Ferrand, L. (2002). Alfred Binet and higher education. History of Psychology, 5, 264-283.

Nicolas, S., \& Gounden, Y. (2016). La psychologie et la psychophysiologie d'Henri Piéron au laboratoire de la Sorbonne. In L. Gutierrez, J. Martin \& R. Ouvrier-Bonnaz (éd.), Henri Piéron (1881-1964). Psychologie, orientation et éducation (pp. 39-62). Toulouse : Octarès. 
Nicolas, S., \& Levine, Z. (2012). Beyond intelligence testing: Remembering Alfred Binet after a century. European Psychologist, 17, 320-325.

Nicolas, S., \& Murray, D. (1999). Théodule Ribot (1839-1916), founder of French psychology: a biographical introduction. History of Psychology, 2, 277-301.

Nicolas, S., Andrieu, B., Croizet, J. C., Sanitioso, R. B., \& Burman, J. T. (2013). Sick? or Slow? On the origins of intelligence as a psychological object. Intelligence, 41(5), 699-711.

Nicolas, S., Andrieu, B., Sanitioso, R. B., Vincent, R., \& Murray, D. J. (2015). Alfred Binet and Crépieux-Jamin: Can intelligence be measured scientifically by graphology? L'Année psychologique / Topic in Cognitive Psychology, 115(1), 3-52.

Nicolas, S., Gras, D., \& Segui, J. (2011). Alfred Binet et le laboratoire de psychologie de la Sorbonne. L'Année psychologique, 111, 293-327.

Nicolas, S., Gounden, Y., \& Sanitioso, R. B. (2014). Alfred Binet, founder of the science of testimony and psycho-legal science. L'Année psychologique / Topic in Cognitive Psychology, 114(2), 209-228.

Nicolas, S., \& Guida, A. (2016). Alfred Binet with Jacques Inaudi: an experimental study of a prodigy of memory. L'Année psychologique / Topic in Cognitive Psychology, 116, 249-293.

Nicolas, S., Gyselinck, V., \& Piolino, P. (2015). Historic note on Henri Piéron's election at the Collège de France (1923). L'Année psychologique / Topic in Cognitive Psychology, 115(2), 177-196.

Nicolas, S., \& Makowski, D. (2016). Can mental fatigue be measured by Weber's compass? Alfred Binet's answer on the value of aesthesiometry (tactile sensitivity) as an objective measure of mental fatigue. European Yearbook of the History of Psychology, 2, pp. 11-46.

Ohayon, A. (1999). L'Impossible Rencontre. Psychologie et psychanalyse en France 1919-1969. Paris : La Découverte.

Piéron, H. (1912). L'œuvre d'Alfred Binet. Revue de psychiatrie et de psychologie expérimentale, $8^{\mathrm{e}}$ série, 16(6), juin, pp. 244-247.

Reuchlin, M. (1955). L'étude scientifique du travail humain : aspects de l'évolution des idées et des méthodes. Journal de psychologie normale et pathologique, 52, 136-155.

Reuchlin, M. (1958). La mesure de l'intelligence, œuvre paradoxale d'Alfred Binet. Bulletin de psychologie, 11, 306-320.

Reuchlin, M. (1965). The historical background for national trends in psychology: France. Journal of the History of the Behavioral Sciences, 1(2), 115-123.

Reuchlin, M. (1966). Histoire de la psychologie ( $5^{\mathrm{e}}$ édition revue et mise à jour). Paris : Presses Universitaires de France (coll. « Que sais-je?»).

Reuchlin, M. (1970). La psychologie différentielle au XIX $x^{\mathrm{e}}$ siècle et au début du Xxe . Métrique statistique et comparaison ordinale. In Actes du XII ${ }^{e}$ Congrès international d'histoire des sciences (Paris, 1968). Texte des rapports (Tome IA, pp. 383-400). Paris : A. Blanchard.

Reuchlin, M. (1971). Naissance de la psychologie appliquée. In M. Reuchlin (éd.), Traité de psychologie appliquée (vol. I, pp. 11-52). Paris : Presses Universitaires de France.

Reuchlin, M. (1984a). Psychologie (5 édition). Paris : Presses Universitaires de France (Fondamental).

Reuchlin, M. (1984b). Histoire de la psychologie (12 édition). Paris : Presses Universitaires de France (coll. «Que sais-je?»). 
Reuchlin, M. (1991a). Histoire de la Psychologie (15édition). Paris : Presses Universitaires de France (coll. «Que sais-je ?»).

Reuchlin, M. (1991b). Bibliographie. Revue européenne de psychologie appliquée, 41(3), 223-227.

Reuchlin, M. (1992). Autobiographie. In F. Parot \& M. Richelle (éd.), Psychologues de langue française (pp. 207-220). Paris : Presses Universitaires de France.

Reuchlin, M. (1995). Totalités, éléments, structures en psychologie. Paris : Presses Universitaires de France.

Reuchlin, M. (2001). Origine et développement du Laboratoire de psychologie différentielle de Paris. Psychologie et Histoire, 2, 59-85.

Reuchlin, M. (2002). Préface. In S. Nicolas, Histoire de la psychologie française (pp. 11-14). Paris : In Press.

Reuchlin, M. (2003a). Contributions à l'histoire des méthodes statistiques employées en psychologie : introduction. Psychologie et Histoire, 4, 1-2.

Reuchlin, M. (2003b). Contributions à l'histoire des méthodes statistiques employées en psychologie : 1. De la régression aux modèles structuraux. Psychologie et Histoire, 4(3), 30. Reuchlin, M. (2003c). Contributions à l'histoire des méthodes statistiques employées en psychologie : 2. Les plans d'expérience et l'analyse de variance : R. A. Fisher (1890-1962). Psychologie et Histoire, 4, 31-60.

Ribot, T. (1881). Les Maladies de la mémoire. Paris : G. Baillière.

Thomas, C., Didierjean, A., \& Nicolas, S. (2016). Scientific study of magic: Binet's pioneering approach based on observations and chronophotography. American Journal of Psychology, 129, 315-328.

Wundt, W. (1907). Ueber Ausfrageexperimente und über die Methoden zur Psychologie des Denkens. Psychologische Studien, 3(4), 301-360.

Wundt, W. (1908). Kritische Nachlese zur Ausfragemethode. Archiv für die Gesamte Psychologie, 11(3-4), 445-459.\#\#10573\#\#

\section{NOTES}

1. . Première édition en $1957 ; 2^{\mathrm{e}}$ éd. en $1959 ; 3^{\mathrm{e}}$ éd. en $1961 ; 4^{\mathrm{e}}$ éd. en $1963 ; 5^{\mathrm{e}}$ édition revue et mise à jour en $1966 ; 6^{\mathrm{e}}$ éd. en $1967 ; 7^{\mathrm{e}}$ édition mise à jour en $1969 ; 8^{\mathrm{e}}$ édition mise à jour en 1972 ; $9^{\mathrm{e}}$ édition mise à jour en $1974 ; 10^{\mathrm{e}}$ éd. en $1978 ; 11^{\mathrm{e}}$ éd. en $1981 ; 12^{\mathrm{e}}$ éd. en $1984 ; 13^{\mathrm{e}}$ édition mise à jour en $1986 ; 14^{\mathrm{e}}$ éd. en $1989 ; 15^{\mathrm{e}}$ édition corrigée en $1991 ; 16^{\mathrm{e}}$ éd. en $1994 ; 17^{\mathrm{e}}$ éd. en 1996 ; $18^{\mathrm{e}}$ éd. en $1999 ; 19^{\mathrm{e}}$ édition mise à jour en $2003 ; 20^{\mathrm{e}}$ édition mise à jour en 2010. Divers retirages ont été réalisés par l'éditeur au cours des 20 dernières années pour s'adapter à la demande (le dernier retirage date de janvier 2012).

2. . On aurait pu aussi prendre en considération les autres chapitres sur la psychologie animale, la psychologie de l'enfant et la psychologie sociale.

3. . Ces auteurs n'ont pas vu en particulier l'intérêt de l'échelle métrique d'intelligence lorsqu'elle fut publiée en 1905 ; pour un historique, voir Nicolas, Andrieu, Croizet, Sanitioso, \& Burman (2013).

4. Souligné par nous. 


\section{RÉSUMÉS}

L'article traite de l'apport de Reuchlin en histoire de la psychologie. L'analyse a porté sur deux de ses principaux écrits : 1) son ouvrage de 1957 sur l'histoire de la psychologie, qui eut de nombreuses rééditions jusqu'en 2012 ; 2) son article sur l'histoire de la psychologie française publié en anglais en 1965. On y montre que Reuchlin a privilégié une histoire objective, mais très personnelle de la psychologie. L'histoire de la psychologie qu'il a proposée est une histoire sousdisciplinaire d'orientation très pédagogique mettant l'accent sur l'orientation scientifique de la discipline. On note cependant beaucoup de manques et des distorsions qui l'ont conduit notamment à sous-estimer l'apport de Binet à la psychologie, alors qu'il s'agit du psychologue français encore le plus connu au monde.

This paper deals with Reuchlin's contribution to the history of psychology. The analysis covers two of his main writings: (1) his 1957 work on the history of psychology, which was republished numerous times up until 2012; (2) his article on the history of French psychology published in English in 1965. We show that Reuchlin favoured an objective but very personal history of psychology. The history of psychology he proposed is a subdisciplinary history, very pedagogical in orientation, which emphasises the scientific orientation of the discipline. However, there are many gaps and distortions that led him, notably, to underestimate Binet's contribution to psychology, even though he is the most well-known French psychologist in the world.

\section{INDEX}

Mots-clés : Reuchlin, histoire de la psychologie, histoire de la psychologie française, psychologie expérimentale, psychologie différentielle, psychologie pathologique

Keywords : Reuchlin, history of psychology, history of French psychology, experimental psychology, differential psychology, pathological psychology

\section{AUTEUR}

\section{SERGE NICOLAS}

est Professeur de psychologie cognitive et d'histoire de la psychologie,

Université Paris Descartes

Membre senior de l'Institut Universitaire de France. Directeur de rédaction de L'Année psychologique. Laboratoire mémoire et cognition, INSERM UMR S894, Université Paris Descartes. Thèmes de recherche : Histoire de la psychologie, épistémologie, psychologie de la mémoire. Contact : Institut de Psychologie, Université Paris Descartes, Laboratoire mémoire et cognition, 71 avenue Édouard-Vaillant, 92774 Boulogne-Billancourt cedex. Courriel : serge.nicolas@parisdescartes.fr 